\title{
Theory of Sound Attenuation in Glasses: The Role of Thermal Vibrations
}

\author{
Jaroslav Fabian ${ }^{1,2}$ and Philip B. Allen ${ }^{2}$ \\ ${ }^{1}$ Department of Physics, University of Maryland at College Park, College Park, Maryland 20742-4111 \\ ${ }^{2}$ Department of Physics and Astronomy, State University of New York at Stony Brook, Stony Brook, New York 11794-3800
}

(Received 13 October 1998)

\begin{abstract}
Sound attenuation and internal friction coefficients are calculated for a realistic model of amorphous silicon. It is found that, contrary to previous views, thermal vibrations can induce sound attenuation at ultrasonic and hypersonic frequencies that is of the same order or even larger than in crystals. The reason is the internal strain induced anomalously large Grüneisen parameters of the low-frequency resonant modes. [S0031-9007(99)08429-X]
\end{abstract}

PACS numbers: $62.65 .+\mathrm{k}, 62.80 .+\mathrm{f}, 63.50 .+\mathrm{x}$

Sound attenuation in glasses is poorly understood. This is because many competing factors lead to sound-wave damping. Most important are thermally activated structural relaxation, hypothetical tunneling states, topological defects, and thermal vibrations. Sorting out different contributions for a given temperature $T$ and sound wave frequency $\nu=\Omega / 2 \pi$ is a difficult task.

Experiments show the following features: (i) At temperatures $T \lesssim 10 \mathrm{~K}$ and ultrasonic frequencies $(10 \mathrm{MHz}$ to $1 \mathrm{GHz}$ ) the sound attenuation coefficient $\Gamma(T)$ exhibits a small, frequency-dependent peak [1]. (ii) At higher temperatures, between 10 and $200 \mathrm{~K}$, another peak in $\Gamma(T)$ develops whose center increases only moderately when $\nu$ increases. The peak broadens at hypersonic frequencies [2] and is not seen above $100 \mathrm{GHz}[3,4]$. As a function of frequency, $\Gamma(\nu) \sim \nu$ at the peak temperatures [2]. (iii) At hypersonic frequencies, $\Gamma(T)$ appears to be almost independent of (or slightly increasing with) $T$ above the peak (ii) to at least $300 \mathrm{~K}[3,4]$. (iv) Room temperature $\Gamma(\nu) \sim \nu^{2}$ from at least $200 \mathrm{MHz}$ [2]; this dependence continues for up to about $300 \mathrm{GHz}[3,4]$ and seems valid for any temperature above the peak (ii). Finally, (v) the attenuation coefficients for longitudinal $\left(\Gamma_{L}\right)$ and transverse $\left(\Gamma_{T}\right)$ waves are similar [2].

While the low-temperature behavior (i) of $\Gamma$ is understood based on the interaction of sound waves with tunneling states [1], features (ii) through (v) lack consistent theoretical justification. The higher-temperature peak (ii) shows many attributes of a thermally activated relaxational process [5], but a calculation shows that to fit experiment, different sets of relaxational processes are needed for different $\nu$ [2]. Also the plateau region (iii) is difficult to explain by a thermally activated relaxation process since numerical fits require unphysically large attempt frequencies [4]. Further, thermal relaxation processes give attenuation that increases more slowly than quadratic with increasing $\nu$, contradicting (iv). Thermal vibrations have been overlooked as a sound-wave damping factor on grounds that vibrational modes would need unreasonably large Grüneisen parameters $(\gamma \approx 200$ for vitreous silica [2]) to account for the measured $\Gamma$. Un- til now, however, there has been no numerical study to test this argument.

In this paper we examine the role that thermal vibrations play in the sound attenuation in glasses. We will use the term "vibron" to refer to any quantized vibrational mode in a glass [6]. Our analysis is restricted to the region $\Omega \tau_{\text {in }} \lesssim 1$ (the so-called Akhiezer regime [7]), where $\tau_{\text {in }}$ is the inelastic lifetime or thermal equilibration time of a thermal vibron. We show that the unusually strong coupling (measured by Grüneisen parameters $\gamma$ ) between sound waves and the low-frequency resonant modes explains the features (iii) through (v). As for the interpretation of (ii), our calculation shows that confusion arises because there actually are two different peaks. One is caused by relaxational processes (not addressed here) and dominates below $1 \mathrm{GHz}$ and another is due to thermal vibrations and dominates at the lowest hypersonic frequencies. A double peak structure should be expected at intermediate frequencies. There is some indication for such structure in measurements on vitreous silica [2]. Our calculation is also a prediction: The existing measurements on amorphous $\mathrm{Si}$ [8] report $\Gamma$ at too low frequencies (300 MHz) to see contributions of thermal vibrations. But even at higher frequencies (say, $30 \mathrm{GHz}$ ) one may expect traces of thermally activated peaks due to various defects. Recently discovered amorphous $\mathrm{Si}$ with 1 at. \% $\mathrm{H}$ [9] in which tunneling (and perhaps also relaxational) processes are inhibited would be excellent to test our results.

In the Akhiezer regime a sound wave passing through a solid can be attenuated by two processes [10]. First, if the wave is longitudinal, periodic contractions and dilations in the solid induce a temperature wave via thermal expansion. Energy is dissipated by heat conduction between regions of different temperatures. Second, dissipation occurs as the gas of vibrons tries to reach an equilibrium characterized by a local (sound-wave-induced) strain. This is the internal friction mechanism. To establish the relative importance of the two processes, consider orderof-magnitude formulas $\Gamma_{h} \approx\left(\Omega^{2} / \rho v^{3}\right)\left(\kappa T \alpha^{2} \rho^{2} v^{2} / C^{2}\right)$ and $\Gamma_{i} \approx\left(\Omega^{2} / \rho v^{3}\right)\left(C T \tau_{\text {in }} \gamma^{2}\right)$ for the heat conductivity and internal friction processes, respectively [10]. Here 
$\rho$ is density, $C$ is specific heat per unit volume, $v$ is sound velocity, $\kappa$ is thermal conductivity, and $\alpha$ is the coefficient of thermal expansion. The ratio $\Gamma_{h} / \Gamma_{i} \approx$ $\left(\kappa \alpha^{2} \rho^{2} v^{2}\right) /\left(C^{3} \tau_{\text {in }} \gamma^{2}\right)$ becomes more intuitive when putting $\alpha \approx C \gamma / B\left(B \approx \rho v^{2}\right.$ is the bulk modulus $)$ and $\kappa \approx C D$, where $D$ is diffusivity. Then $\Gamma_{h} / \Gamma_{i} \approx$ $D /\left(v^{2} \tau_{\text {in }}\right)$. The factor $v^{2} \tau_{\text {in }}$ measures the ability of vibrons to absorb energy from a sound wave of velocity $v$. The difference between a glass and a crystal lies in the values of $D$ and $\tau_{\text {in }}$. In crystals $D \approx v^{2} \tau_{\text {in }}$, that is, energy is carried by phonon wave packets with group velocity $v$. The ratio $\Gamma_{h} / \Gamma_{i}$ is then of order unity. In glasses energy is transferred by diffusion (spreading rather than ballistic propagation of wave packets [11]) and $D$ is not related to $\tau_{\text {in }}$ [12]. One of the reasons the contribution to $\Gamma_{i}$ of thermal vibrons was previously underestimated is that $\tau_{\text {in }}$ was guessed from thermal conductivity [2]; this gave too small $\tau_{\text {in }}$. For amorphous $\mathrm{Si}, D \approx 10^{-6} \mathrm{~m}^{2} / \mathrm{s}[12], v \approx$ $8 \times 10^{3} \mathrm{~m} / \mathrm{s}$, and $\tau_{\text {in }} \approx 10^{-12} \mathrm{~s} \mathrm{[6]} \mathrm{give} \Gamma_{h} / \Gamma_{i} \approx 0.02$. Since these are typical values, $\Gamma_{h}$ can be neglected. This is consistent with experiment: Compared with crystals, glasses have smaller $\kappa$ and yet $\Gamma$ can be larger [2].

Internal friction leads to sound-wave energy attenuation [10] $\Gamma=\left(\Omega^{2} / \rho v^{3} q^{2}\right) \eta_{\alpha \beta \gamma \delta} q_{\alpha} e_{\beta} q_{\gamma} e_{\delta}$, where $\eta_{\alpha \beta \gamma \delta}$ is the internal friction tensor with cartesian coordinates $\alpha, \ldots, \delta$ and $\mathbf{q}$ (e) is the wave vector (polarization) of the sound wave. Summation over repeated indices is assumed. We will evaluate $\Gamma$ for both longitudinal $(L)$ and transverse $(T)$ sound waves with wave vectors averaged over all directions:

$$
\begin{aligned}
& \Gamma_{L}=\frac{\Omega^{2}}{15 \rho v_{L}^{3}}\left(\eta_{\alpha \alpha \beta \beta}+2 \eta_{\alpha \beta \alpha \beta}\right), \\
& \Gamma_{T}=\frac{\Omega^{2}}{30 \rho v_{T}^{3}}\left(3 \eta_{\alpha \beta \alpha \beta}-\eta_{\alpha \alpha \beta \beta}\right) .
\end{aligned}
$$

The coefficients $\eta_{\alpha \beta \gamma \delta}$ are the real part of a complex tensor $\bar{\eta}_{\alpha \beta \gamma \delta}$ which can be obtained by solving a kinetic equation in relaxation time approximation [7],

$\bar{\eta}_{\alpha \beta \gamma \delta}=\sum_{j} T c_{j} \tau_{j} \frac{\gamma_{\alpha \beta}^{j} \gamma_{\gamma \delta}^{j}-\left(\bar{\gamma}_{\alpha \beta} \gamma_{\gamma \delta}^{j}+\gamma_{\alpha \beta}^{j} \bar{\gamma}_{\gamma \delta}\right) / 2}{1-i \Omega \tau_{j}}$.

The summation is over all vibrational modes $j ; c_{j}$ and $\tau_{j}$ denote mode specific heat and relaxation time. The Grüneisen tensor $-\gamma_{\alpha \beta}^{j}$ is the relative shift of mode frequency $\omega_{j}$ per unit strain $\epsilon_{\alpha \beta} ; \bar{\gamma}$ is the mode average of $\gamma^{j}$ weighted with $c_{j} /\left(1-i \Omega \tau_{j}\right)$. The applicability of kinetic theory to the problem of internal friction was justified by DeVault and co-workers [13] who obtained $\eta$ from a microscopic theory as an autocorrelation function of the momentum current density operator. Remarkably, the microscopic theory shows that the momentum current in a solid is not monopolized by ballistically propagating vibrational modes as in the case of the energy current. Nonpropagating (even localized) modes can contribute as much as propagating ones to the momentum current. One consequence is that the concept of "minimum kinetic coefficient," as introduced for electrical [14] or heat [15] conductivity of disordered systems, is not realized for internal friction. We generalized [16] DeVault's theory to include internal strain, the atomic rearrangements in a strained solid. We found that internal strain affects internal friction only by modifying $\gamma^{j}$, as in the case of thermal expansion [17]: $\gamma^{j}$ now reflects the change between the initial mode frequency and the frequency of the mode after the rescaling (scaling parameter $1+\epsilon$ ) plus the rearranging of atomic positions (to achieve a new equilibrium at strain $\epsilon$ ). Internal strain is very important for thermal expansion of glasses [17]; we will show that it is important for $\eta$ (and $\Gamma$ ) as well.

We calculate $\eta$ and $\Gamma$ for the model of amorphous $\mathrm{Si}$ based on the Wooten-Winer-Weaire atomic coordinates [18] and Stillinger-Weber interatomic forces [19], with 1000 atoms arranged in a cube of side $27.549 \AA$ with periodic boundary conditions. Diagonal Grüneisen parameters $\gamma_{\alpha \alpha}^{j} / 3 \equiv\left(\gamma_{11}^{j}+\gamma_{22}^{j}+\gamma_{33}^{j}\right) / 3$ for this model [20] were given in Ref. [17]; transverse $\gamma_{\alpha \beta}$ are calculated here. Vibrational lifetimes $\tau_{j}$ are extracted from their 216-atom version values [6] (see also Ref. [21]). The model has sound velocities $v_{L}=7640 \mathrm{~m} / \mathrm{s}$ and $v_{T}=$ $3670 \mathrm{~m} / \mathrm{s}$ [22].

Figure 1 shows the calculated $\Gamma(\nu)$ for longitudinal and transverse sound waves in amorphous Si from $10 \mathrm{MHz}$ to $1 \mathrm{THz}$ at $300 \mathrm{~K}$. The attenuation $\Gamma \sim \nu^{2}$ up to about $100 \mathrm{GHz}$, where the condition for the applicability of kinetic theory $\Omega \tau_{\text {in }} \lesssim 1$ reaches its limit $\left(\tau_{\text {in }} \approx 1 \mathrm{ps}\right)$. Our calculation is not valid beyond this point. In comparison, the measured attenuation of longitudinal waves in vitreous silica grows quadratically with $\nu$ up to at least $400 \mathrm{GHz}$

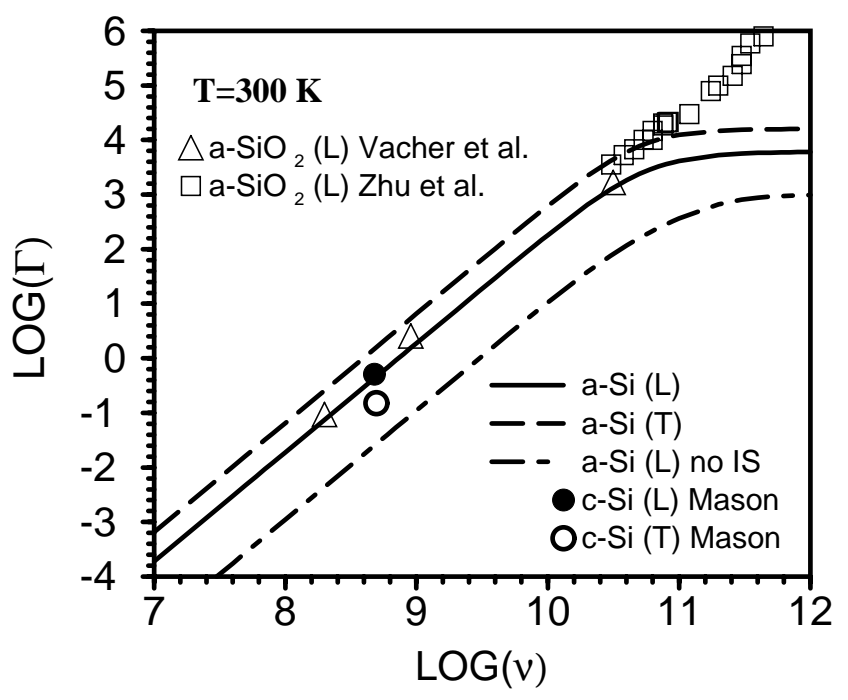

FIG. 1. Log-log plot of the sound attenuation $\Gamma\left(\mathrm{cm}^{-1}\right)$ at 300 $\mathrm{K}$, as a function of sound-wave frequency $\nu(\mathrm{Hz})$. Calculated $\Gamma$ are represented by lines (IS: internal strain), experimental data by symbols. L (T) stands for longitudinal (transverse) sound waves. 
[4], suggesting that $\tau_{\text {in }}$ in vitreous silica is several times smaller than in amorphous $\mathrm{Si}$. This is not surprising since $\mathrm{Si}$ is remarkably harmonic: Room temperature heat conductivity of crystalline $\mathrm{Si}$ is larger by an order of magnitude than that of quartz [23], and a similar relation may hold for the corresponding $\tau_{\text {in }}$ of the glassy phases.

More surprising is the comparison with crystalline Si. Figure 1 shows that $\Gamma_{L}$ is similar for the amorphous and crystalline cases (measured $\Gamma$ for vitreous silica is several times larger than for quartz [2]). One would naively expect the sound attenuation in a glass to be much smaller than in the corresponding crystal since, owing to a distribution of bond lengths and bond angles, anharmonicity of the glass is higher (and $\tau_{\text {in }}$ smaller). The same interatomic potential, for example, yields $\tau_{\text {in }}$ for high-frequency phonons in crystalline $\mathrm{Si}$ at $300 \mathrm{~K}$ about 5 times larger than in amorphous Si [6]. The reason why $\Gamma$ in glasses can be of the same order or even higher than in crystals is the internal-strain-induced anomalously large Grüneisen parameters of the resonant modes [17] (see also Fig. 3 below). (Resonant modes are low-frequency extended modes whose amplitudes are unusually large at a small, typically undercoordinated region [17,24].) Atomic rearrangements caused by internal strain are largest in the same regions of undercoordination where the resonant modes have largest amplitude [17]. This leads to high sensitivity (measured by $\gamma$ ) of the frequencies of these modes to strain. If the internal strain is neglected, the sound attenuation is an order of magnitude smaller, as seen in Fig. 1. (Since the resonant modes have low frequencies, their $\tau_{j}$ is longer than an average $\tau_{\text {in }}$; this adds even more weight to these modes.) Fewer than $1 \%$ of the modes are capable of increasing $\Gamma$ by a decade. We believe the measured $\Gamma$ for vitreous silica is also caused by the strong coupling of sound waves and resonant modes. Vitreous silica is a much more open structure than amorphous Si so the number of resonant modes should be higher, bringing $\Gamma$ above the crystalline value.

Another interesting feature in Fig. 1 is the relative attenuation strength for longitudinal and transverse sound waves. While our model of amorphous $\mathrm{Si}$ gives $\Gamma_{L} / \Gamma_{T} \approx$ $1 / 3$ at $300 \mathrm{~K}$, the measured ratio for crystalline $\mathrm{Si}$ is reversed: $\Gamma_{L} / \Gamma_{T} \approx 3$ [23]. This again shows how differently is sound attenuated in glasses and in crystals. The ratio $\Gamma_{L} / \Gamma_{T}$ can be written as $\left(v_{T} / v_{L}\right)^{3}\left(\gamma_{L}^{2} / \gamma_{T}^{2}\right)$, where $\gamma_{L}$ and $\gamma_{T}$ are effective Grüneisen parameters. A crude way to estimate $\gamma_{L}^{2}$ and $\gamma_{T}^{2}$, suggested by Eqs. (1) and (2), is to take mode averages of $\left(\gamma_{\alpha \alpha}^{j} \gamma_{\beta \beta}^{j}+2 \gamma_{\alpha \beta}^{j} \gamma_{\alpha \beta}^{j}\right) / 15$ and $\left(3 \gamma_{\alpha \beta}^{j} \gamma_{\alpha \beta}^{j}-\gamma_{\alpha \alpha}^{j} \gamma_{\beta \beta}^{j}\right) / 30$. Our model gives $\gamma_{L}^{2} \approx$ 3 and $\gamma_{T}^{2} \approx 1$. The ratio $\Gamma_{L} / \Gamma_{T}$ is then about $1: 3$, in accord with the full calculation. Assuming the same ratio $\gamma_{L}^{2} / \gamma_{T}^{2} \approx 3$ for vitreous silica $\left(v_{L}=5800 \mathrm{~m} / \mathrm{s}\right.$ and $v_{T}=3800 \mathrm{~m} / \mathrm{s}$ ), transverse and longitudinal waves are attenuated about equally. This is observed in experiment [2]. The explanation of the measured $\Gamma_{L} / \Gamma_{T}$ in crystalline Si can be found in Ref. [23].
In Fig. 2 we plot $\Gamma(T)$ for different $\nu$. A remarkable feature is a peak at about $20 \mathrm{~K}$ at $1 \mathrm{MHz}$ and below. As $\nu$ increases, the peak shifts towards higher $T$ and vanishes above $4-5 \mathrm{GHz}$. Two factors cause the peak. (a) The $\operatorname{sum} \sum_{j} c_{j}\left(\gamma^{j}\right)^{2}$ saturates at much lower temperatures (about $50 \mathrm{~K}$ ) than the model Debye temperature $T_{D} \approx$ $450 \mathrm{~K}$ [22]. This is because the relevant $j$ are resonant modes with small frequencies. (b) For low-frequency modes, $T \tau_{j}$ (after increasing linearly) develops a peak, before going constant [much like $\Gamma(T)$ itself]. As the temperature dependence of $\Gamma$ follows $\sum_{j} c_{j}\left(\gamma^{j}\right)^{2} T \tau_{j}$, the peak appears. At large $\nu$ the peak vanishes because of the factor $1 /\left(1+\Omega^{2} \tau^{2}\right)$ in Eq. (3). At $T$ above $100 \mathrm{~K}, \Gamma(T)$ is nearly constant, as observed in experiment as a plateau (iii). This again follows from (a) and (b).

We are not aware of any experiment with which we could compare our calculations. The measurement of $\Gamma(T)$ of sputtered amorphous Si films reported in Ref. [8], for example, was performed at $300 \mathrm{MHz}$. This is too low to see any contributions from thermal vibrations. The whole temperature spectrum is dominated by a single peak of the type (ii), except at very low temperatures. This peak is expected to increase linearly with $\nu$, until thermal vibrations become relevant (roughly at $10 \mathrm{GHz}$ ), causing a plateau (iv) that increases as $\nu^{2}$ at higher frequencies. Even at smaller frequencies one may see some vibrational contribution to $\Gamma(T)$ at large enough $T$, since the thermally activated peak decreases as $1 / T$ at large $T$.

Anomalous low $T$ thermal expansion already suggested [25] very large $\gamma$ values for low $\omega$ modes. Our large $\gamma$ values [17] agree nicely with trends in $\alpha(T)$. Like thermal expansion, $\Gamma$ should be strongly sample and model dependent. There is evidence [26] that our highly homogeneous model of amorphous $\mathrm{Si}$ becomes free of resonant modes when the number of atoms grows to infinity. That means

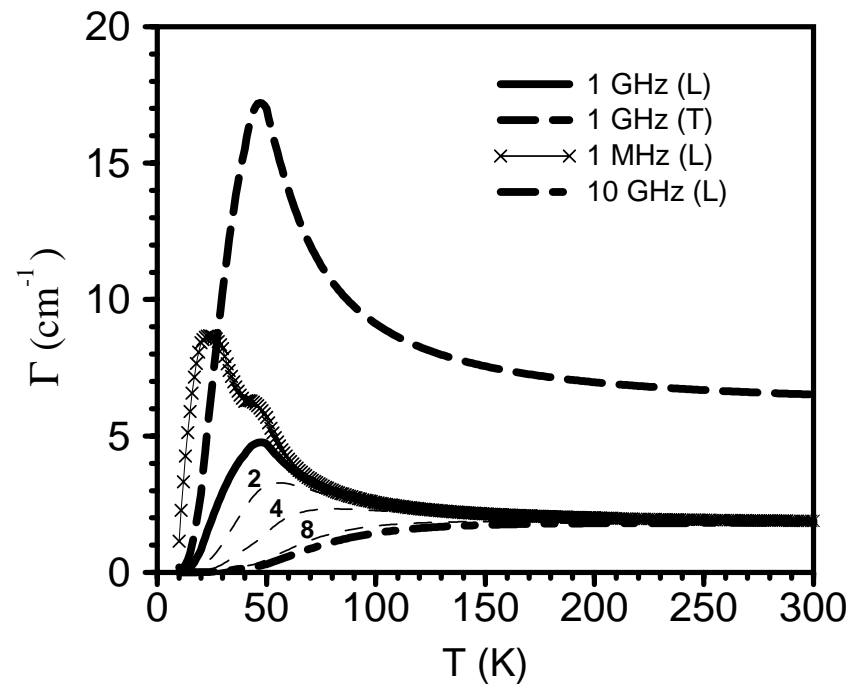

FIG. 2. Calculated sound attenuation $\Gamma(T)$ for amorphous $\mathrm{Si}$ at different frequencies. The thin dashed lines are for longitudinal waves with the labeled $\nu$ in GHz. Plotted are rescaled values $\Gamma / \nu^{2}$ for $\nu$ measured in $\mathrm{GHz}$. 


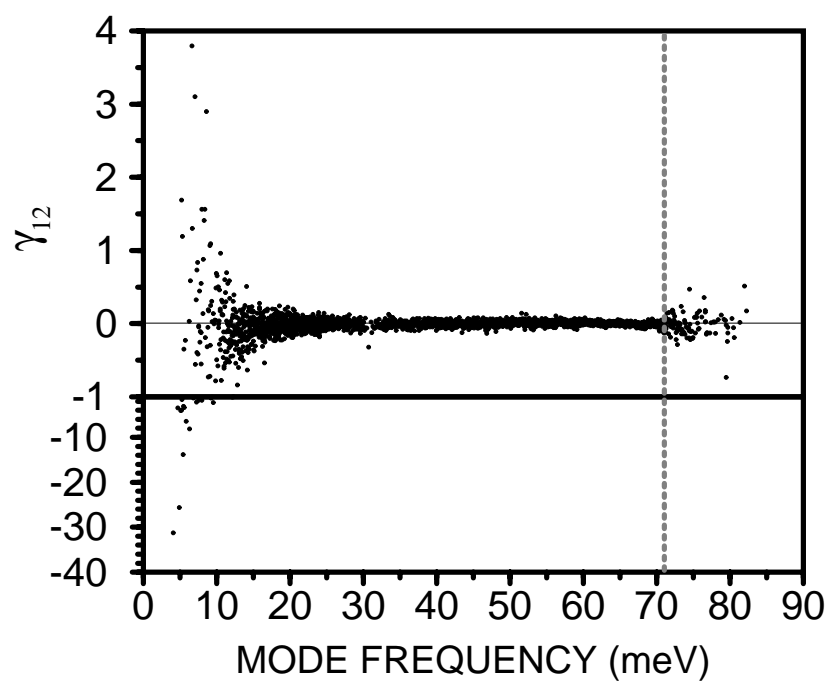

FIG. 3. Calculated transverse Grüneisen parameters $\gamma_{12}$ for amorphous $\mathrm{Si}$ as a function of vibron frequency. Above the vertical line $(\approx 71 \mathrm{meV})$ the modes are localized.

an infinite model would predict $\Gamma$ about a decade smaller than calculated here. Amorphous silicon, however, can be prepared only in thin films, where voids and other inhomogeneities are unavoidable. Voids loosen the strict requirements of a tetrahedral random network (for example, by introducing free boundary conditions). Then, as in our finite models, regions of undercoordinated atoms will allow the formation of resonant modes. While this issue for amorphous silicon will be ultimately settled by experiment, our calculation combined with the existing data on vitreous silica strongly suggests the reality of resonant modes.

Our final note concerns the mode dependence of transverse Grüneisen parameters such as $\gamma_{12}$. Similar to volumetric $\gamma_{\alpha \alpha} / 3$ [17], transverse $\gamma_{12}$ in Fig. $3\left(\gamma_{13}\right.$ and $\gamma_{23}$ look the same) is unusually large for resonant modes and has scattered values for high-frequency localized modes. (More resonant modes have $\gamma_{12}$ negative than positive, which suggests that resonant modes are trapped at highly anisotropic undercoordinated regions whose sizes change under shear [17].) The $15-70 \mathrm{meV}$ vibrons (diffusons [6]) have $\gamma_{12} \approx 0$ (average magnitude 0.02), while the corresponding $\gamma_{\alpha \alpha} / 3$ are of order unity [17]. Such small values (zeros in an infinite model) are characteristic for diffusons, which are extended modes whose polarization directions (atomic displacements) point, in general, at random. There remains only a short-range correlation between polarization directions which determines the diffuson's frequency $\omega_{d}$. If a shear, say, $\epsilon_{12}$, is applied, $\omega_{d}$ changes to $\omega_{d}^{\prime}\left(\epsilon_{12}\right)$. Since long-range order in the diffuson polarization is absent, $\omega_{d}^{\prime}\left(\epsilon_{12}\right) \approx \omega_{d}^{\prime}\left(-\epsilon_{12}\right)$, and $\gamma_{12}$ which is a linear coefficient in the expansion of $\omega_{d}^{\prime}$ in $\epsilon_{12}$ must vanish.

We thank J.L. Feldman for helpful discussions. The work was supported by NSF Grant No. DMR 9725037. J.F. also acknowledges support from the U.S. ONR.
[1] S. Hunklinger and W. Arnold, in Physical Acoustics, edited by W.P. Mason and R.N. Thurston (Academic, New York, 1976), p. 155.

[2] R. Vacher, J. Pelous, F. Plicque, and A. Zarembowitch, J. Non-Cryst. Solids 45, 397 (1981).

[3] C. J. Morath and H.J. Maris, Phys. Rev. B 54, 203 (1996).

[4] T. C. Zhu, H. J. Maris, and J. Tauc, Phys. Rev. B 44, 4281 (1991); H. N. Lin, R. J. Stoner, H. J. Maris, and J. Tauc, J. Appl. Phys. 69, 3816 (1991).

[5] D. Tielbürger, R. Merz, R. Ehrenfels, and S. Hunklinger, Phys. Rev. B 45, 2750 (1992).

[6] J. Fabian and P.B. Allen, Phys. Rev. Lett. 77, 3839 (1996).

[7] H. J. Maris, in Physical Acoustics, edited by W.P. Mason and R.N. Thurston (Academic, New York, 1971), Vol. VIII.

[8] M. Von Haumeder, U. Strom, and S. Hunklinger, Phys. Rev. Lett. 44, 84 (1980).

[9] X. Liu, B.E. White, R. O. Pohl, E. Iwanizcko, K. M. Jones, A. H. Mahan, B.N. Nelson, R.S. Crandall, and S. Veprek, Phys. Rev. Lett. 78, 4418 (1997).

[10] V.L. Gurevich, Transport in Phonon Systems (NorthHolland, Amsterdam, 1986).

[11] P.B. Allen and J. Kelner, Am. J. Phys. 66, 497 (1998).

[12] P. B. Allen and J. L. Feldman, Phys. Rev. B 48, 12581 (1993); J. L. Feldman, M.D. Kluge, P.B. Allen, and F. Wooten, Phys. Rev. B 48, 12589 (1993).

[13] G. P. DeVault and J. A. McLennan, Phys. Rev. 138, A856 (1965); G. P. DeVault, Phys. Rev. 149, 624 (1966); 155, 875 (1967); G. P. DeVault and R. J. Hardy, Phys. Rev. 155, 869 (1967).

[14] N. F. Mott, Philos. Mag. 22, 7 (1970).

[15] C. Kittel, Phys. Rev. 75, 972 (1949).

[16] J. Fabian, Ph.D. thesis, SUNY, Stony Brook, NY, 1997.

[17] J. Fabian and P.B. Allen, Phys. Rev. Lett. 79, 1885 (1997).

[18] F. Wooten, K. Winer, and D. Weaire, Phys. Rev. Lett. 54, 1392 (1985).

[19] F. H. Stillinger and T. A. Weber, Phys. Rev. B 31, 5262 (1985).

[20] Reference [17] used a slightly different version with a small amount of residual stress. The main results, however, remain unchanged. J.L. Feldman (private communication).

[21] S. R. Bickham and J. L. Feldman, Phys. Rev. B 57, 12234 (1998); Philos. Mag. B 77, 513 (1998).

[22] J. L. Feldman, J. Q. Broughton, and F. Wooten, Phys. Rev. B 43, 2152 (1991).

[23] W.P. Mason, in Physical Acoustics, edited by W.P. Mason (Academic, New York, 1965), Vol. III.

[24] R. Biswas, A. M. Bouchard, W. A. Kamikatahara, G. S. Grest, and C. M. Soukoulis, Phys. Rev. Lett. 60, 2280 (1988); H. R. Schober and B. Laird, Phys. Rev. B 44, 6746 (1991); H. R. Schober and C. Oligschleger, Phys. Rev. B 53, 11469 (1996).

[25] G. K. White, Phys. Rev. Lett. 34, 204 (1975).

[26] J. L. Feldman, P.B. Allen, and S. R. Bickham (unpublished). 